Research Article

\title{
Isotropic Gravastar Model in Rastall Gravity
}

\author{
G. Abbas $\mathbb{D}$ and K. Majeed \\ Department of Mathematics, The Islamia University of Bahawalpur, Bahawalpur, Pakistan \\ Correspondence should be addressed to G. Abbas; ghulamabbas@iub.edu.pk
}

Received 26 September 2020; Revised 28 October 2020; Accepted 4 November 2020; Published 16 November 2020

Academic Editor: Yu Liu

Copyright (c) 2020 G. Abbas and K. Majeed. This is an open access article distributed under the Creative Commons Attribution License, which permits unrestricted use, distribution, and reproduction in any medium, provided the original work is properly cited.

\begin{abstract}
In the present paper, we have introduced a new model of gravastar with an isotropic matter distribution in Rastall gravity by the Mazur-Mottola (2004) mechanism. Mazur-Mottola approach is about the construction of gravastar which is predicted as an alternative to black hole. By following this convention, we define gravastar in the form of three phases. The first one is an interior phase which has negative density; the second part consists of thin shell comprising ultrarelativistic stiff fluid for which we have discussed the length, energy, and entropy. By the graphical analysis of entropy, we have shown that our proposed thin shell gravastar model is potentially stable. The third phase of gravastar is defined by the exterior Schwarzschild geometry. For the interior of gravastar, we have found the analytical solutions free from any singularity and the event horizon in the framework of Rastall gravity.
\end{abstract}

\section{Introduction}

For the very first time, in the 18th century, Laplace and Michell gave the clues of existence of a celestial body having extreme density and strongest gravitational effect enough that even the light cannot be escaped. Later, celestial body in outer space was termed as "black hole" by American physicist Wheeler with the development of general relativity in 1916. In the review of black hole, it has three layers: the inner and outer event horizons and the singularity. The event horizon is the boundary of the black hole which lies between the inside and outside of black hole where the gravitational pull is so strong that nothing can escape even the light. However, the singularity is known as the center of a black hole, a single point which has huge mass of black hole in concise nearly around zero volume. It is hypothesized by astronomers that this singular point is hidden behind event horizon where all laws of physics fail due to its infinite density and gravity. A lot of work has been done on black holes by the researchers [1-6]. With the various successive study on black holes, the astronomers endure two main problems of black holes, i.e., event horizon and singularity. To avoid singularity, regular black hole is the better choice than classical black holes, but the event horizon problem remained unresolved [7-9].
It was required to formulate such an alternative system that could tackle the abovementioned challenges; then, in 2001, Mazur and Mottola [10,11] suggested a marvelous idea of a gravitational vacuum star termed as gravastar that looks like a black hole, in other words, "a substitution of black hole". With the extension of Bose-Einstein condensation concept at low temperature, they $[10,11]$ have designed a hypothetical model of an isotropic interior de Sitter spacetime as cold, dark, and compact objects in such a way that it can be tackled without facing the problems of event horizon and any singularity. However, the exterior of gravastar is completely vacuum, which can be explained by Schwarzschild geometry. The center of gravastar is known as dark energy, and the boundary is the thin shell which isolates its interior and exterior regions. Thermodynamically, the Mazur and Mottola idea is stable, and it has become one of the most attractive debates in the present decade. The gravastar configuration consists of three different parts which can be distinguished with the values of equations of state parameter, and the three regions of a gravastar are labeled as follows: I, interior region: $0<r<R_{1}$ with EOS $p=-\rho$; II, thin shell: $R_{1}<r<R_{2}$ with EOS $p=\rho$; and III, exterior region: $r>R_{2}$ at $p=\rho=0$, where $p$ and $\rho$ represent 
isotropic pressure and matter density and $R_{1}$ and $R_{2}$ represent the interior and exterior radii of the gravastar, respectively. The thickness of thin shell can be calculated by $\epsilon=R_{2}-R_{1}$ which is very small, i.e., $0<\epsilon \ll 1$ but finite. The most interesting and impressive point in the construction of gravastar is that it required exact solutions which are regular in their entire domain. Many researchers have done more work on the gravastar with great interest to obtain singularity-free solution [12-19].

In 2011, Usmani et al. [20] provided an exact solution for internally charged gravastar with conformal motion by taking Reissner-Nordström as an exterior spacetime. On vanishing the charge from gravitational mass equation in the interior region, the total gravitational mass shifted into electromagnetic mass under some specific conditions and this aspect assures that interior de Sitter vacuum of a charged gravastar must induce the gravitational mass. This model encouraged the other researchers to do more work on gravastar. So, in 2014, Bhar [21] generalized a study of Usmani et al. [20] to higher dimensional Reissner-Nordström spacetime. All the physical aspects of this study matches to the results of Usmani et al. [20]. After this, Bhar [22] provided another model on charged gravastar with conformal motion in a modified theory, i.e., $f(T)$ gravity. All the solutions for an isotropic pressure, charged density, and electric field have been found with the help of linear function, i.e., $f(T)=a T+b$. Here, again the physical features match with the results of Usmani et al. [20]. The study of Usmani et al. [20] has been reviewed again in another modified theory, i.e., $f(R, T)$ gravity by Sharif et al. [23]. The expression $f(R, T)=R+2 \beta T$ has been used in this model, and all the exact, singularity-free solutions with conformal Killing vector approach were found. By substituting $\beta=0$, all the results of Usmani et al. [20] can be recovered.

Rahaman et al. [24] investigated a model of gravastars for three-dimensional anti-de Sitter spacetime with negative cosmological constant. Later on, Rahaman et al. [25] extended their own work [24] with the addition of an electric charge. With the influence of charge distribution, they [25] found out all the exact solutions and physical features. Ghosh et al. [26] have presented a solution of gravastar for $D$-dimensions with a charge distribution, and actually, it was the generalized work of Rahaman et al. [25]. The results were stable and singularity-free gravastar. Later on, Ghosh et al. [27] have explored the exact, stable, and nonsingular results of gravastar without an electric charge for higher dimensional de Sitter spacetimes with cosmological constant $\Lambda$. Recently, the gravastar model for four dimensions with the implementation of Karmarkar condition in GR has been introduced by Ghosh et al. [28]. The shell results have been obtained without thin shell approximation and put forward the stable model which looks like black hole. Das et al. [29] have investigated the exact solutions of gravastar in $f(R, T)$ gravity. In this model, pressure has been defined by negative matter density in the interior region, and thin shell has been supposed to be filled with ultrarelativistic fluid, taking the exterior region as completely vacuum and defined by de Sitter Schwarzschild spacetime. By following these assumptions, exact, nonsingular, and nonevent horizon solutions have been found which highlight the stability and validity of gravastar. In $f(G, T)$ theory of gravity, the gravastar model has been constructed by Shamir et al. [30]. Collection of all exact solutions and physical features with graphical representation made their model as stable, event horizon free, and nonsingular, which can be regarded as an alternative to black hole. The electromagnetic effects on gravastar taking spherical symmetric spacetime in $f(T)$ theory of gravity have been examined by Debnath [31]. In this study, two cases have been discussed: (i) $T=0$ and (ii) $f_{T T}=0$. It was concluded that gravastar cannot be constructed for the $T=0$ case. But in case of $f_{T T}=0$, all analytical solutions were obtained for three regions: I, interior; II, thin shell; and III, exterior, and provided a stable model of gravastar. Riessner-Nordström solutions have been achieved in the exterior region rather than Schwarzschild spacetime. Recently, Ghosh et al. [32] investigated the new solutions of gravastar by taking Einstein field equations in $f(\mathbb{T}, \mathscr{T})$ gravity. By using modified action in terms of torsion scalar $\mathbb{T}$ and trace of energy momentum tensor $\mathscr{T}$, the nonsingular solutions consist of pressure and density for interior, thin shell, junction condition, and the physical parameters have been obtained which indicate the stability of gravastar. Currently, a new model of gravastar with electromagnetic field in the framework of $f(R, T)$ theory of gravity by using the Mazur-Mottola $[10,11]$ methodology has been provided by Majeed et al. [33]. They [33] analyzed the role of electric charge on the entire analytical solutions which are stable, nonevent horizon and singularity free in nature. Also, Yousaf and his collaborators [34-38] have worked on the stability of gravastars in several modified theories of gravity.

In cosmological point of view, general relativity (GR) acts as a pillar to understand the stellar structures with concept of cosmological constant which is responsible to control accelerated expansion universe. Despite the great discovery of GR, there has been an intense need of some modifications because of having numerous problems related to universe expansion. In addition, GR has a world famous component, that is, covariant conservation of energy momentum tensor. Consequently, the total mass of a system remains conserved in general theory of relativity, but it is not proved experimentally. So, some modified theories have been proposed by astronomers which are inattentive about covariant conservation of energy momentum tensor. In 1972, Rastall [39] nominated a modified theory with some modifications in law of conservation of energy momentum tensor in GR. Rastall transforms the conservation law of energy momentum tensor in GR (i.e., $T_{\tau ; \sigma}^{\tau}=0$ ) to the nonconservation law (i.e., $T_{\tau ; \sigma}^{\tau}=\zeta R_{, \sigma}$ ) by introducing the coupling parameter $\zeta$. This parameter is taken to be the measure of tendency of the geometry to couple with matter field in a nonminimal way. Metaphysically, Rastall theory gravity figures out the impacts of quantum fields in a curved spacetime in a covariant manner. These mentioned fundamental and cosmological aspects of Rastall theory motivate us to explore the analytical solutions in the framework of this theory. 
Recently, Rastall theory has been reviewed extensively by many researchers [40-46]. Debnath [47] has explored the solutions of gravastars in Rastall-Rainbow gravity. He used the charge distribution as a function of Rastall's parameter and Rainbow function, and the exact and nonsingular solutions have been obtained. In this study, the physical properties depend on Rastall parameter and Rainbow function. Salako et al. [44] have discussed the stability of anisotropic compact stars in Rastall theory by using Krori and Barua static spherically symmetric spacetime. Abbas and Shahzad [48] have analyzed the solutions of an isotropic compact stars by using conformal Killing vector and EOS parameter $\omega$ which is limited as $0<\omega<1$, in Rastall theory. In this study, the variety of physical properties has been discussed such as isotropic behavior of compact stars, nonconservation equation, energy conditions, surface redshift, and stability of the model. In other work by Shahzad and Abbas [49], they have investigated the solutions for three different anisotropic compact stars, i.e., $4 U 1820-30$, Her $X-1$, and SAXJ $1808.4-3658$ with radii $10 \mathrm{~km}, 7.7 \mathrm{~km}$, and $7.07 \mathrm{~km}$, respectively.

Despite the attractive aspects of the Rastall theory, recently, Visser [50] urged that Rastall theory is equivalent to GR. In response to Visser paper [50], Darabi et al. [51] proved that both theories are entirely different and the Visser claim was wrong explicitly. The important point of the Visser argument [50] was the definition of the energy momentum tensor (EMT) given by Rastall was not correct, and Rastall's proposal is just the rearrangement of the matter sector of the GR, whereas Darabi et al. [51] argued that the definition of the EMT in Rastall's proposal is in agreement with the usual definition of EMT. To support their argument, they provided a compatible example of $f(R)$ theory of gravity by using Visser's procedure [50], and after some calculations, they proved that $f(R)$ theory is also equivalent to the $G R$, which is incorrect.

In the present study, we have investigated some physical aspects of gravastar in Rastall theory of gravity and found the analytical solutions for different phases of gravastar structure. The paper is designed in the following pattern. Section 2 consists of review of Rastall theory with its Einstein field equations and nonconservation law of energy momentum tensor. In Section 3, the gravastar structure has been discussed by three different regions, i.e., (a) interior region, (b) thin shell, and (c) exterior region as well as the junction conditions. We examine the physical aspects, in particular, proper length, energy, entropy, and EOS in Section 4. In the last section, we conclude our results.

\section{Field Equations and Their Solutions in Rastall Theory}

By modifying the conservation law of energy momentum tensor in GR (i.e., $T_{\tau ; \sigma}^{\tau}=0$ ), Rastall tendered a nonconservation theory of gravity; according to this theory, the nonconservation law is

$$
T_{\tau ; \sigma}^{\tau}=\zeta R_{, \sigma},
$$

where $\zeta$ is the Rastall parameter which shows the digression from GR as well as the relation between matter field and geometry. Following this modified theory, the developed field equations can be expressed as

$$
G_{\tau \sigma}+\kappa \zeta g_{\tau \sigma} R=\kappa T_{\tau \sigma}
$$

From the above equation, $\kappa, R$, and $T_{\tau \sigma}$ are Rastall coupling constant, Ricci scalar, and energy momentum tensor, respectively. The field equations for the present theory can be redesigned as

$$
G_{\tau \sigma}=\kappa\left(T_{\tau \sigma}-\frac{\zeta \kappa g_{\tau \sigma T}}{4 \kappa \zeta-1}\right)
$$

The energy momentum tensor for an isotropic fluid is

$$
T_{\tau \sigma}=(\rho+p) g_{\tau \sigma}-p g_{\tau \sigma}
$$

Now, we consider a static spherically symmetric line element in $(3+1)$ dimensions for the interior spacetime as

$$
\mathrm{d} s^{2}=e^{\mu(r)} \mathrm{d} t^{2}-e^{\nu(r)} \mathrm{d} r^{2}-r^{2} \mathrm{~d} \theta^{2}-r^{2} \sin ^{2} \theta \mathrm{d} \phi^{2},
$$

where $\mu(r)$ and $\nu(r)$ be the metric functions. In the association of matter distribution (5) and energy momentum tensor (4), the Einstein-Maxwell field equations in Rastall gravity are

$$
\begin{aligned}
-1+e^{\nu}+r \nu^{\prime} & =r^{2} e^{\nu} \kappa\left(\rho-\frac{\kappa \zeta(\rho-3 p)}{4 \kappa \zeta-1}\right),(6) \\
1-e^{\nu}+r \mu^{\prime} & =r^{2} e^{\nu} \kappa\left(p+\frac{\kappa \zeta(\rho-3 p)}{4 \kappa \zeta-1}\right),(7) \\
\frac{r^{2}}{4}\left(2 \mu^{\prime \prime}+\mu^{\prime 2}-\mu^{\prime} \nu^{\prime}\right)+\frac{r}{2}\left(\mu^{\prime}-\nu^{\prime}\right) & =r^{2} e^{\nu} \kappa\left(p+\frac{\kappa \zeta(\rho-3 p)}{4 \kappa \zeta-1}\right) .
\end{aligned}
$$

According to Rastall's modification (i.e., from equation (1)), the nonconservation law of energy momentum tensor can be written in the following form:

$$
\frac{8 \pi \zeta}{32 \pi \zeta-1} \frac{\mathrm{d}}{\mathrm{d} r}(\rho-3 p)-\frac{\mathrm{d} p}{\mathrm{~d} r}-\frac{\nu^{\prime}}{2}(\rho+p)=0
$$

\section{Gravastar Construction}

To construct a gravastar in Rastall theory, we discuss three regions, which are described as follows.

3.1. Interior Region. In this section, we shall find the solutions for the interior region of gravastar. For this purpose, we first assume the following form of equation of state:

$$
p=-\rho \text {. }
$$

With the help of equations (9) and (10), one can get

$$
p=-\rho=-c_{0} \text {, }
$$


where $c_{0}$ is the constant matter density throughout the interior region. By using equations (6) and (11), we can find the metric potential as

$$
e^{-v}=1+\frac{r^{2}}{3} \kappa\left(\frac{4 \kappa \lambda c_{0}}{4 \kappa \lambda-1}-c_{0}\right)+\frac{c_{1}}{r},
$$

where $c_{1}$ shows the constant of integration. For regular solution at center $(r=0)$, we set $c_{1}$ equal to zero and obtain

$$
e^{-v}=1+\frac{r^{2}}{3} \kappa\left(\frac{4 \kappa \lambda c_{0}}{4 \kappa \lambda-1}-c_{0}\right) .
$$

The relation between $\mu$ and $\nu$ with the help of equations (6), (7), and (11) can be obtained as

$$
e^{\mu}=c_{2} e^{-v}
$$

where $c_{2}$ is the constant of integration. As mentioned above, the matter density remains constant throughout the interior region (see equation (11)); thus, we can find the active gravitational mass in the following form:

$$
M(C)=\int_{0}^{R_{1}=C} 4 \pi r^{2} c_{0} \mathrm{~d} r=\frac{4}{3} \pi C^{3} c_{0},
$$

which is the mass contained in the interior region of radius $R_{1}=C$.

3.2. Thin Shell. The shell is the middle region of interior and exterior, which consists of ultrarelativistic perfect fluid with high density, obeying the $\operatorname{EOS} p=\rho$. By applying $p=\rho$, it is more difficult to determine the exact solutions. In order to avoid this difficulty, we take the thin shell approximation, i.e., $0<e^{-\nu} \equiv h \ll 1$, and try to investigate the analytical results. With the implementation of this approximation, we reconstruct Rastall's field equations (6)-(8), by setting $h$ as zero in association with $\operatorname{EOS}(p=\rho)$, which can be achieved in the following form:

$$
\begin{aligned}
1-r h^{\prime} & =r^{2} \kappa\left(\rho+\frac{2 \kappa \zeta \rho}{4 \kappa \zeta-1}\right), \\
-1 & =r^{2} \kappa\left(\rho-\frac{2 \kappa \zeta \rho}{4 \kappa \zeta-1}\right), \\
\frac{r h^{\prime}}{2}+\frac{\mu^{\prime} h^{\prime}}{4} r^{2} & =r^{2} \kappa\left(\rho-\frac{2 \kappa \zeta \rho}{4 \kappa \zeta-1}\right) .
\end{aligned}
$$

By solving the above field equations, one can get the following results:

$$
\begin{aligned}
e^{-v} & =\ln \left|\left(c_{3} r^{1+\alpha}\right)\right| \\
e^{\mu} & =c_{4} e^{-2 r(1+r / 1+\alpha)},
\end{aligned}
$$

where

$$
\alpha=\frac{1+2 \kappa \zeta / 4 \kappa \zeta-1}{1-2 \kappa \zeta / 4 \kappa \zeta-1},
$$

and $c_{3}$ and $c_{4}$ are the constants of integration. In the consequence of equation (9) and $\operatorname{EOS} p=\rho$, we have

$$
\rho=p=e^{\left(c_{4}+c_{5}\right) \beta}\left(e^{-2 r(1+r / 1+\alpha) \beta}\right),
$$

where $c_{5}$ is a constant of integration and $\beta=32 \pi \zeta-1 / 1-48 \pi \zeta$.

3.3. Exterior Spacetime and Junction Conditions. The static Schwarzschild geometry in the exterior region with EOS $(p=\rho=0)$ can be expressed as

$$
\mathrm{d} s^{2}=\left(1-\frac{2 M}{r}\right) \mathrm{d} t^{2}-\left(1-\frac{2 M}{r}\right)^{-1} \mathrm{~d} r^{2}-r^{2}\left(\mathrm{~d} \theta^{2}+\sin \theta^{2} \mathrm{~d} \phi^{2}\right) .
$$

Here, $M$ indicates the total mass of the gravastar. The gravastar structure is divided into three zones, i.e., (i) interior, (ii) shell, and (iii) exterior. The interior zone connects with exterior at a junction interface, i.e., $r=C$. After the review of Darmois-Israel [52, 53], the metric functions should be continuous at the junction surface (i.e., $r=C$ ) for the smooth matching between the gravastar zones (i) and (iii). Thus, the stress-energy surface tensor at the junction can be defined in the following form:

$$
S_{\alpha \beta}=-\frac{1}{8 \pi}\left(\kappa_{\alpha \beta}-\delta_{\alpha \beta} \kappa_{\gamma \gamma}\right)
$$

where $\kappa_{\alpha \beta}=K_{\alpha \beta}^{+}-K_{\alpha \beta}^{-}$and signs + and - indicate exterior and interior regions, respectively. The second elemental form linked with two sides of thin shell is given by

$$
K_{\alpha \beta}^{ \pm}=-\left.n_{\sigma}^{ \pm}\left(\frac{\partial^{2} x_{\sigma}}{\partial \phi^{\alpha} \partial \phi^{\beta}}+\Gamma_{i j}^{\sigma} \frac{\partial x^{i}}{\partial \phi^{\alpha}} \frac{\partial x^{j}}{\partial \phi^{\beta}}\right)\right|_{\Omega} .
$$

In the above equation, the intrinsic coordinates on shell expressed by $\phi^{\alpha}$ and $n_{\sigma}^{ \pm}$are symbolized for two-sided unit normals to surface $\Omega$ which can be stated individually as

$$
n_{\sigma}^{ \pm}= \pm\left|g^{i j} \frac{\partial f}{\partial x^{i}} \frac{\partial f}{\partial x^{j}}\right|^{-1 / 2} \frac{\partial f}{\partial x^{\sigma}}
$$

with $n^{\lambda} n_{\lambda}=1$. By following the Lanczos approach [52], we can find surface stress-energy tensor as $S_{\alpha \beta}=\operatorname{diag}[\varepsilon,-\vartheta,-\vartheta,-\vartheta]$ defining $\varepsilon$ as surface energy density and $\vartheta$ as the surface pressure. These two parameters $\varepsilon$ and $\vartheta$ have the following form:

$$
\begin{aligned}
& \varepsilon=-\frac{1}{4 \pi C}[\sqrt{f}]_{-}^{+} \\
& \vartheta=-\frac{\varepsilon}{2}+\frac{1}{16 \pi}\left[\frac{f^{\prime}}{\sqrt{f}}\right]_{-}^{+} .
\end{aligned}
$$

Equations (27) and (28) give the following results:

$$
\begin{aligned}
& \varepsilon=-\frac{1}{4 \pi C}\left(\sqrt{1-\frac{2 M}{C}}-\sqrt{1+\frac{\kappa}{3}\left(\frac{4 \kappa \zeta c_{0}}{4 \kappa \zeta-1}-c_{0}\right) C^{2}}\right), \\
& \vartheta=\frac{1}{8 \pi C}\left[\frac{1-M / C}{\sqrt{1-2 M / C}}-\frac{1+2 / 3 \kappa\left(4 \kappa \zeta c_{0} / 4 \kappa \zeta-1-c_{0}\right) C^{2}}{\sqrt{1+\kappa / 3\left(4 \kappa \zeta c_{0} / 4 \kappa \zeta-1-c_{0}\right) C^{2}}}\right] .
\end{aligned}
$$


The mass of thin shell is calculated as

$$
m_{\mathrm{sh}}=4 \pi C^{2} \varepsilon=C\left[\sqrt{1+\frac{\kappa}{3}\left(\frac{4 \kappa \zeta c_{0}}{4 \kappa \zeta-1}-c_{0}\right) C^{2}}-\sqrt{1-\frac{2 M}{C}}\right] .
$$

By manipulating equation (31), one can get the following form of total mass present in the stellar structure:

$$
M=\frac{\kappa}{6}\left(c_{0}-\frac{4 \kappa \zeta c_{0}}{4 \kappa \zeta-1}\right) C^{2}-\frac{m_{s}^{2}}{2 C}+m_{s} \sqrt{1+\frac{\kappa}{3}\left(\frac{4 \kappa \zeta c_{0}}{4 \kappa \zeta-1}-c_{0}\right) C^{2}}
$$

\section{Some Physical Properties}

4.1. Proper Length and Energy within the Shell. The proper thickness of the shell connects the interior region to the exterior at $r=C$ and $r=C+\epsilon$. It is taken to be very small as $0<\epsilon \ll 1$ and can be determined as

$$
l=\int_{C}^{C+\epsilon} \sqrt{e^{\nu}} \mathrm{d} r=\int_{C}^{C+\epsilon} \frac{1}{\sqrt{\ln \left(c_{3} r^{1+\alpha}\right)}}=\left[\frac{\sqrt{\pi} r \operatorname{Erfi}\left(\sqrt{\log \left(r^{1+\alpha} c_{3}\right)} / \sqrt{1+\alpha}\right)\left(r^{1+\alpha} c_{3}\right)^{-1 / 1+\alpha}}{\sqrt{1+\alpha}}\right]_{C}^{C+\epsilon} .
$$

Now, we calculate the energy within the shell as

$$
\begin{aligned}
& E=\int_{C}^{C+\epsilon} 4 \pi r^{2} \rho \mathrm{d} r=4 \pi \int_{C}^{C+\epsilon} r^{2} e^{\left(c_{4}+c_{5}\right) \beta} e^{-2 r(1+r / 1+\alpha) \beta} \mathrm{d} r, \\
& =\left[\begin{array}{c}
\frac{2(1+\alpha) e^{\beta\left(c_{4}+c_{5}-2 r(1+\alpha+r) / 1+\alpha\right)} \pi \sqrt{\beta}(1+\alpha-2 r)}{4 \beta^{3 / 2}}+ \\
\frac{(1+\alpha)^{3 / 2} \sqrt{2 \pi}(1+\beta+\alpha \beta) e^{\beta\left(c_{4}+c_{5}-2 r(1+\alpha+r) / 1+\alpha\right)} e^{\beta(1+\alpha+2 r)^{2} \beta / 2(1+\alpha)} \operatorname{Erf}((1+\alpha+2 r) \sqrt{\beta} / \sqrt{2(1+\alpha)})}{4 \beta 3 / 2}
\end{array}\right]_{C}^{C+\epsilon} \\
& s(r)=\frac{\alpha^{2} k_{B}^{2} T(r)}{4 \pi \hbar^{2}}=\alpha\left(\frac{k_{B}}{\hbar}\right) \sqrt{\frac{p}{2 \pi}},
\end{aligned}
$$
which is stable for the single condensate area. On the contrary, the entropy within the shell can be found by the following formula:

$$
S=\int_{C}^{C+\epsilon} 4 \pi r^{2} s(r) \sqrt{e^{v}} \mathrm{~d} r
$$

where $s(r)$ is the energy density, which can be represented as where $\alpha$ is considered as a constant. In this present study, use $G=c=1$ and $k_{B}=\hbar=1$ and then

$$
s(r)=\alpha \sqrt{\frac{p}{2 \pi}} \text {. }
$$
result
Finally, the usage of equations (19), (22), and (35) gives 


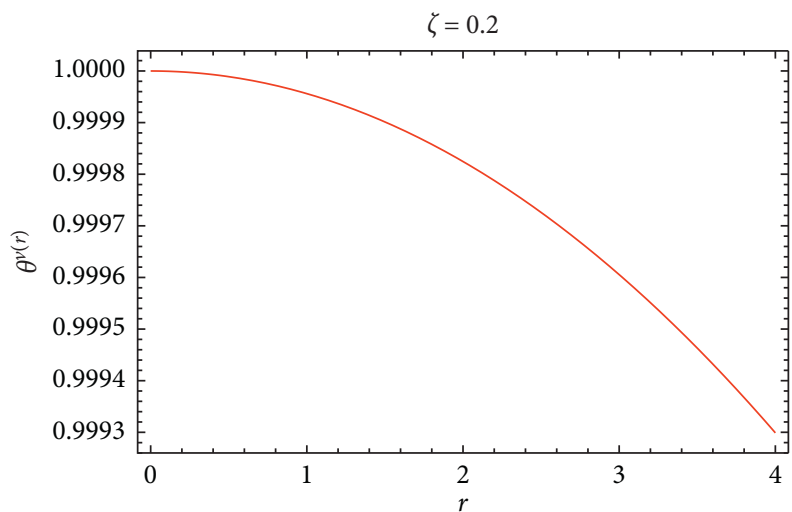

FIGURE 1: Variation in metric potential within the interior region with respect to the radial coordinate $r$.

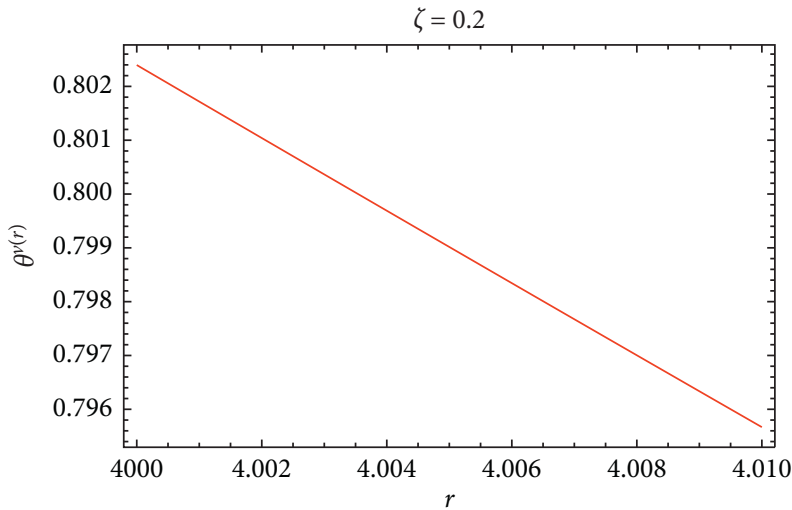

Figure 2: Variation in metric potential $e^{\nu(r)}$ within the shell with respect to radial coordinate $r$.

$$
\begin{aligned}
& S=\frac{4 \pi \xi}{\sqrt{2 \pi}} \int_{C}^{C+\epsilon}\left[r^{2} \sqrt{\left.\frac{e^{\left(c_{4}+c_{5}\right) \beta} e^{-2 r(1+r / 1+\alpha) \beta}}{\ln \left(c_{3} r^{1+\alpha}\right)}\right]} d r,\right. \\
& S=\frac{4 \pi \xi}{\sqrt{2 \pi}} \int_{C}^{C+\epsilon} \frac{\mathrm{d} f(r)}{\mathrm{d} r} \mathrm{~d} r=\frac{4 \pi \xi}{\sqrt{2 \pi}}[f(r)]_{C}^{C+\epsilon}=\frac{4 \pi \xi}{\sqrt{2 \pi}}[f(C+\epsilon)-f(C)] .
\end{aligned}
$$

4.3. Equation of State. By using equations (29) and (30) in the expression $\vartheta=\omega(C) \mathcal{\varepsilon}$, the EoS parameter at $r=C$ can be obtained in the following form:

$$
\omega(C)=\frac{\left[1-M / C / \sqrt{1-2 M / C}-1+2 / 3 \kappa\left(4 \kappa \zeta c_{0} / 4 \kappa \zeta-1-c_{0}\right) C^{2} / \sqrt{1+\kappa / 3\left(4 \kappa \zeta c_{0} / 4 \kappa \zeta-1-c_{0}\right) C^{2}}\right]}{2\left[-\sqrt{1-2 M / C}+\sqrt{1+\kappa / 3\left(4 \kappa \zeta c_{0} / 4 \kappa \zeta-1-c_{0}\right) C^{2}}\right]} .
$$

In the above equation, one can note that there are different terms as well as square root terms that increase the sensitivity of EOS parameter, i.e., $\omega(C)$. For such a situation, it demands some restrictions which are $2 M / C<1$ and $\kappa / 3\left(c_{0} / 1-4 \kappa \zeta\right)<1$ to maintain $\omega(C)$ as real. However, with the implementation of binomial series up to the 1st order on the square root terms in equation (39) subject to the constraints $M / C \ll 1$ and $\kappa / 3\left(c_{0} / 1-4 \kappa \zeta\right) \ll 1$, one can obtain the approximation

$$
\omega(C) \approx \frac{3 / 2}{\left[6 M / \kappa\left(c_{0} / 1-4 \kappa \zeta\right) C^{3}-1\right]} .
$$

By following the above approximation, there are two probabilities: the parameter $\omega(C)$ will be positive for 


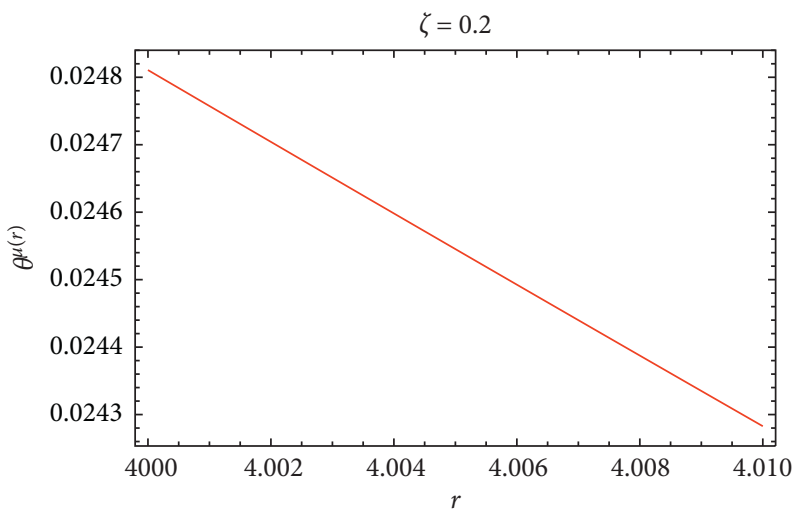

FIgURE 3: Variation in metric potential $e^{\mu(r)}$ within the shell with respect to radial coordinate $r$.

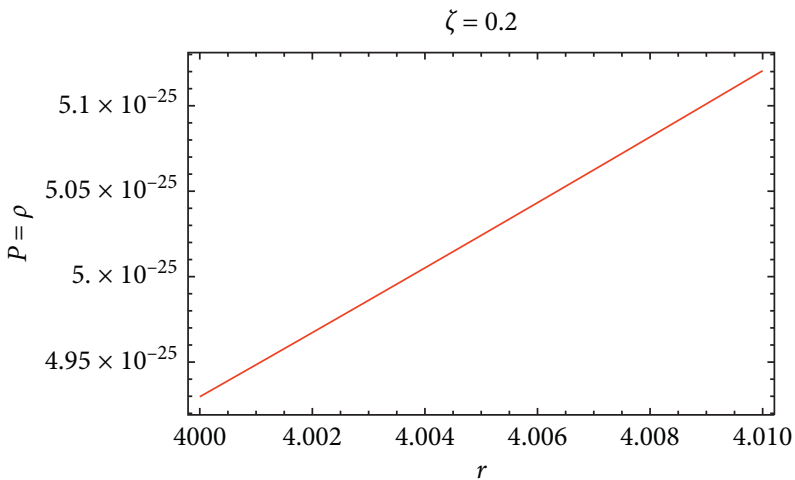

FIGURE 4: Variation in isotropic pressure $p=\rho\left(\mathrm{km}^{-} 2\right)$ within the shell with respect to radial coordinate $r$.

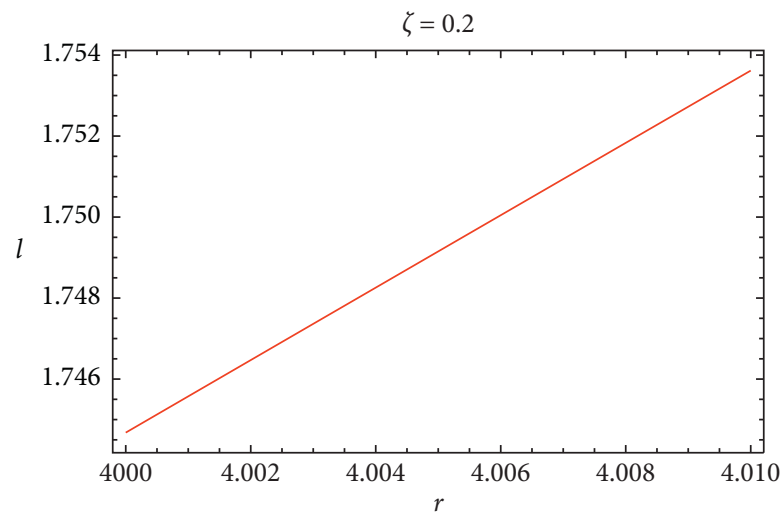

FiguRE 5: Variation in proper length $(\mathrm{km})$ of thin shell with respect to radial coordinate $r$.

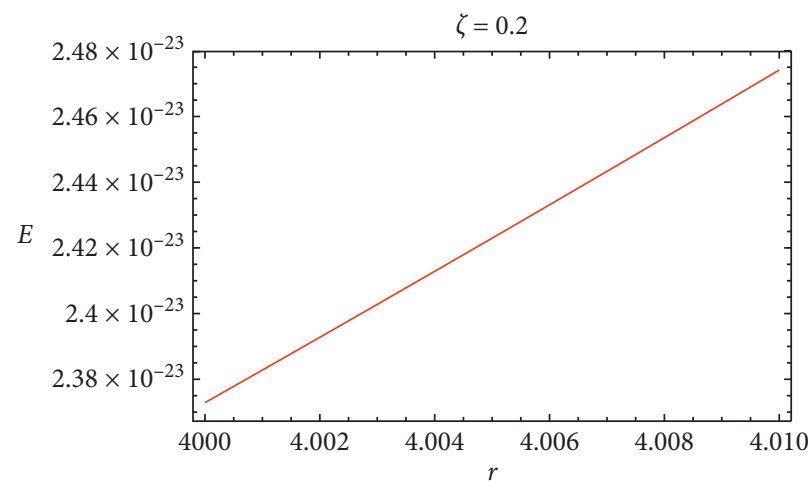

Figure 6: Variation in energy within the shell with respect to radial coordinate $r$. 


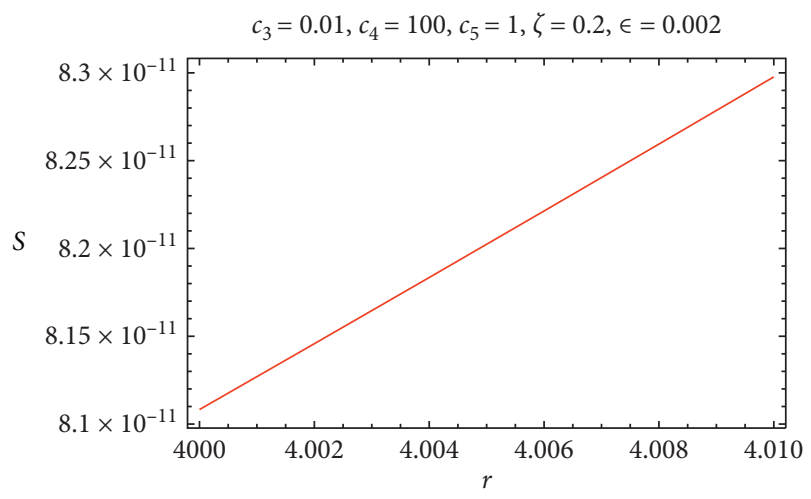

FIGURE 7: Variation in entropy within the shell of with respect to radial coordinate $r$.

condition $M / C^{3}>\kappa / 6\left(c_{0} / 1-4 \kappa \zeta\right) C^{3}$ and $\omega(C)$ will be negative for $M / C^{3}>\kappa / 6\left(c_{0} / 1-4 \kappa \zeta\right) C^{3}$. In view of above discussion, some cases arise: (i) if $\omega(C)=-1$, it gives the cosmological constant and predicts dark energy; (ii) $\omega(C)<-1$ informs us about the hypothetical phantom energy; (iii) when $\omega(C)<-1 / 3$, this situation indicates the accelerated expansion of universe and the case $\omega(C)>-1$ is for nonphantom.

\section{Conclusion}

In the present paper, we have analyzed the isotropic fluid new exact solutions of gravastar by considering spherically symmetric and static spacetime in the context of Mazur and Mottola [1, 2] in the Rastall theory of gravity. The main advantage of working on gravastar is that it seems as black hole, but it resolves most of the problems related to black hole. In the present work, we have studied the effects of nonconservation of energy-momentum tensor on the solutions of gravastar by the use of Rastall's field equations. In the present investigation, we get all the results free from singularity and event horizon in the influence of Rastall's parameter throughout the gravitating system (i.e., interior region, shell and shell properties, and exterior region). In the light of Mazur and Mottola [1, 2] concept, the gravastar layout consists of three regions: $\mathrm{I}$, interior region $\left(0 \leq r<r_{1}=C\right)$; II, shell $\left(C=r_{1}<r<r_{2}=C+\epsilon\right)$; III, exterior $\left(r>r_{2}=C+\epsilon\right)$. The physical significance of these regions is given as follows:

(i) Interior region (I): it is enclosed by a thin shell made with ultrarelativistic stiff fluid and is defined by negative isotropic pressure with positive energy density, i.e., $p=-\rho$, and this pressure generates the repulsive force over the thin shell. The central density denoted as $c_{0}$ has been retrieved with the help of nonconservation law equation (9), which gives us information about pressure and density remaining constant throughout the interior region. We obtained the results for pressure, density, and the metric potentials by dealing with equations
(6)-(8). All these results for interior region of gravastar are regular at origin $r=0$. The physical behavior for metric potential $e^{v}$ with respect to the radial coordinate $r$ is been shown in Figure 1. From this physical interpretation, it can be shown that the metric potential remains positive, regular at $r=o$, finite and free from central singularity. The active gravitational mass also has the same properties.

(ii) Thin shell (II): the shell of gravastar with $\operatorname{EOS} p=\rho$ is assumed to be very thin formed with ultrarelativistic matter distribution bonding with the cold baryonic universe called stiff fluid with very high density. To find the exact solutions in the shell region with EOS, $p=\rho$ for gravastar is very difficult, so we have used the thin shell approximation as $0<e^{-v} \equiv h \ll 1$ and get modified form of Rastall's field equations, i.e., equations (16)-(18). Through these equations, we have found exact solutions of metric potentials (i.e., $e^{\mu}$ and $e^{\nu}$ ) and matter density within the shell. Figures 2 and 3 show the physical behavior with positive metric potentials $e^{\nu(r)}$ and $e^{\mu(r)}$, respectively. However, Figure 4 demonstrates the increasing and positive profile of energy density within the shell. These outcomes help us to deal with shell properties such as proper length, energy, and entropy obtained by using equations (19) and (22). The positive, linearly increasing and finite pattern throughout the shell for shell properties (i.e., length, energy, and entropy) is shown in Figures 5-7. All these graphical representations reveal the physical authenticity of our stellar structure (i.e., gravastar). In cosmology, the EOS parameter $\omega$ is established for isotropic fluids by the ratio of pressure $p$ and density $\rho$. This parameter gives different values for different choice of parameters. For example, it has value -1 in case of cosmological constant, and less than -1 leads to the phantom energy.

(iii) Exterior region (III) and junction conditions: with EOS $p=\rho=0$, the exterior region is defined by Schwarzschild spacetime which can be seen in equation (23). At the junction interface, the interior region joins with the exterior region with smooth matching at $r=C$. In accordance with Darmois-Israel conditions $[1,2]$, we have derived some aspects like surface energy density, surface pressure, mass of thin shell, and the mass of gravastar which are given in equations (29)-(32).

In the end, we arrive at conclusion that we have found a new exact solution which are regular and finite at origin. This is physically acceptable condition for gravastar in Rastall's theory of gravity. Moreover, we have obtained a nonsingular and event horizon-free model but seems to be an alternative to black hole in this present study. These points fulfil the requirements which are necessary for the existence of gravastar. On the contrary, traditional black holes of 
Einstein's theory are nonregular and infinite at origin and have event horizons at finite values of radius $r$. For example, Schwarzschild black hole has event horizon at $r=2 \mathrm{~m}$, which lies inside the photon sphere of radius $r=3 \mathrm{~m}$. The constraints resulting from interferometric LIGO detectors GW150914 on the compactness of the merging objects are in accordance with the large compactness that can be linked with gravastars. On the basis of these investigations, one cannot exclude the possibility of inspiral signal because of gravastars. In view of this, it has been predicted by Chirenti and Rezzolla [54] that the merged object would be a rotating gravastar. We would like to mention that this work can be extended in other nonconservative and matter-curvature coupled modified theories of gravity [55].

\section{Data Availability}

The data that support the findings of this study are available from the corresponding author upon reasonable request.

\section{Conflicts of Interest}

The authors declare that they have no conflicts of interest.

\section{References}

[1] Mukhopadhyay, A., and Singh, P., 2003, http://arxiv.org/abs/ $0301002 \mathrm{v} 3$.

[2] Ashtekar, A., Olmedo, J. and Singh, P., 2018, http://arxiv.org/ abs/1806.00648v3.

[3] A. Ditta and G. Abbas, "Relativistic accretion mechanism for some black holes," Chinese Journal of Physics, vol. 65, p. 325, 2020.

[4] D. Gregoris and K. Rosquist, "Observational backreaction in discrete black holes lattice cosmological models," 2020, http:// arxiv.org/abs/arXiv:2006.00855v1.

[5] A. Sánchez, "Geometrothermodynamics of black holes with a nonlinear source," 2020, http://arxiv.org/abs/2006.00023v1.

[6] K. A. Meissner and H. Nicolai, "Supermassive gravitinos and giant primordial black holes," 2020, http://arxiv.org/abs/2007. $11889 \mathrm{v} 1$

[7] J. Bardeen, "Australian shipping and passenger records," in Proceedings of GR5, Sydney, Australia, 1968.

[8] A. Borde, "Open and closed universes, initial singularities, and inflation," Physical Review D, vol. 50, p. 3392, 1994.

[9] A. Borde, "Regular black holes and topology change," Physical Review D, vol. 55, no. 12, p. 7615, 1997.

[10] P. Mazur and E. Mottola, "Gravitational condensate stars: an alternative to black holes," Report Number. LA-UR-01-5067, 2001.

[11] P. O. Mazur and E. Mottola, "Gravitational vacuum condensate stars," Proceedings of the National Academy of Sciences, vol. 101, no. 26, p. 9545, 2004.

[12] R. Chan, "Stability analysis of lower dimensional gravastars in non commutative geometry," Journal of Cosmology and Astroparticle Physics, vol. 1110, p. 013, 2011.

[13] R. Chan, M. F. A. Da Silva, and P. Rocha, "Gravastars and black holes of anisotropic dark energy," General Relativity and Gravitation, vol. 43, no. 8, p. 2223, 2011.

[14] M. P. Moruno, "Bounce and cyclic cosmology in extended nonlinear massive gravity," Journal of Cosmology and Astroparticle Physics, vol. 1203, p. 034, 2012.
[15] F. Lobo, "Linearized stability analysis of gravastars in noncommutative geometry," Journal of High Energy Physics, vol. 1312, p. 065, 2013.

[16] N. Sakai, "Wave optics in spacetimes with compact gravitating object," Physical Review D, vol. 90, Article ID 104013, 2014.

[17] T. Kubo and N. Sakai, "Gravitational lensing by gravastars," Physical Review D, vol. 93, Article ID 084051, 2016.

[18] A. Banerjee, J. R. Villanueva, P. Channuie, and K. Jusufi, "Stable gravastars: guilfoyle's electrically charged solutions," Chinese Physics C, vol. 42, p. 11, Article ID 115101, 2018.

[19] R. Chan and M. F. A. D. Silva, "How the charge can affect the formation of gravastars," Journal of Cosmology and Astroparticle Physics, vol. 2010, no. 7, p. 29, 2010.

[20] A. A. Usmani, F. Rahaman, S. Ray et al., "Charged gravastars admitting conformal motion," Physics Letters B, vol. 701, no. 4, p. 388, 2011.

[21] P. Bhar, "Higher dimensional charged gravastar admitting conformal motion," Astrophysics and Space Science, vol. 354, 2014.

[22] P. Bhar, "A new model of charged gravstar in $f(T)$ gravity admitting conformal motion," 2017, http://arxiv.org/abs/ $1702.02467 \mathrm{v} 1$.

[23] M. Sharif and A. Waseem, "Charged gravastars with conformal motion in $f(R, T)$ gravity," 2019, http://arxiv.org/abs/ 1911.08316v1.

[24] F. Rahaman, S. Ray, A. A. Usmani, and S. Islam, "The higher dimensional gravastars," Physics Letters B, vol. 707, p. 319, 2011.

[25] F. Rahaman, S. Ray, A. A. Usmani, and S. Islam, "The (2+1)dimensional charged gravastars," Physics Letters B, vol. 717, p. 1, 2012

[26] S. Ghosh, F. Rahaman, B. K. Guha, and S. Ray, "Charged gravastars in higher dimensions," Physics Letters B, vol. 767, p. 380, 2017.

[27] G. Ghosh, S. Ray, F. Rahaman, and B. K. Guha, "Gravastars with higher dimensional spacetimes," 2018, http://arxiv.org/ abs/1701.01046v3.

[28] S. Ghosh, S. Biswas, F. Rahaman, B. K. Guha, and S. Ray, "Gravastars in $(3+1)$ dimensions admitting Karmarkar condition," Annals of Physics, vol. 411, Article ID 167968, 2019.

[29] A. Das, G. Ghosh, and B. K. Guha, "Gravastars in $f(R, T)$ gravity," Physical Review D, vol. 95, Article ID 124011, 2017.

[30] M. F. Shamir and M. Ahmad, "Gravastars in $f(R, G)$ gravity," Physical Review D, vol. 97, Article ID 104031, 2018.

[31] U. Debnath, "Charge gravastars in $f(T)$ modified gravity," European Physical Journal C, vol. 79, p. 499, 2019.

[32] S. Ghosh, A. D. Kanfon, A. Das, M. J. S. Houndjo, I. G. Salako, and S. Ray, "Gravastars in $f(\mathbb{T}, \mathscr{I})$ gravity," International Journal of Modern Physics A, vol. 35, no. 4, Article ID 2050017, 2020.

[33] K. Majeed, Z. Yousaf, Z. Yousaf, and G. Abbas, "Effects of electromagnetic field on the stability of locally isotropic gravastars," New Astronomy, vol. 80, Article ID 101397, 2020.

[34] Z. Yousaf, M. Z. Bhatti, and T. Naseer, "General relativity and quantum cosmology," Physics of the Dark Universe, vol. 28, Article ID 400535, 2020.

[35] Z. Yousaf, M. Z. Bhatti, and U. Farwa, "Role of $f(R, T, R \mu \nu T \mu \nu)$ model on the stability of cylindrical stellar model," European Physical Journal C, vol. 77, p. 359, 2017, http://arxiv.org/abs/ 1705.06975 .

[36] Z. Yousaf, K. Bamba, and M. Z. Bhatti, "Influence of modification of gravity on the dynamics of radiating spherical 
fluids," Physical Review D, vol. 93, no. 6, Article ID 064059, 2016.

[37] Z. Yousaf, K. Bamba, and M. Z. Bhatti, "Causes of irregular energy density in $f(R, T)$ gravity," Physical Review D, vol. 93, Article ID 124048, 2016.

[38] Z. Yousaf, K. Bamba, M. Z. Bhatti, and U. Ghafoor, "Charged gravastars in modified gravity," Physical Review D, vol. 100, 2019 http://arxiv.org/abs/1907.05233, Article ID 024062.

[39] P. Rastall, "Generalization of the Einstein theory," Physical Review D, vol. 6, no. 12, p. 3357, 1972.

[40] G. Abbas and M. R. Shahzad, "A new model of quintessence compact stars in the rastall theory of gravity," The European Physical Journal A, vol. 54, p. 211, 2018.

[41] M. R. Shahzad and G. Abbas, "Strange stars with MIT bag model in the Rastall theory of gravity," International Journal of Geometric Methods in Modern Physics, vol. 16, no. 9, Article ID 1950132, 2019.

[42] G. Das, "Einstein-Yang-Mills AdS black brane solution in massive gravity and viscosity boun," European Physical Journal C, vol. 78, p. 810, 2018.

[43] G. Abbas and M. R. Shahzad, "Comparative analysis of Einstein gravity and Rastall gravity for the compact objects," Chinese Journal of Physics, vol. 63, p. 1, 2020.

[44] I. G. Salako, A. Jawad, and H. Moradpour, "Anisotropic compact stars in non-conservative theory of gravity," International Journal of Geometric Methods in Modern Physics, vol. 15, no. 6, Article ID 1850093, 2018.

[45] K. Lin and L.-W. Qian, "Neutral regular black hole solution in generalized Rastall gravity," Chinese Phys. C, vol. 43, Article ID 083106, 2019.

[46] R. Li, J. Wang, Z. Xu, and X. Guo, "Constraining the Rastall parameters in static space-times with galaxy-scale strong gravitational lensing," Monthly Notices of the Royal Astronomical Society, vol. 486, no. 2, p. 2407, 2019.

[47] U. Debnath, "Charged gravastars in rastall-rainbow gravity," 2019, http://arxiv.org/abs/1909.01139v1.

[48] G. Abbas and M. R. Shahzad, "Models of anisotropic compact stars in the Rastall theory of gravity," Astrophysics and Space Science, vol. 363, p. 251, 2018.

[49] G. Abbas and M. R. Shahzad, "Models of anisotropic compact stars in the Rastall theory of gravity," Astrophysics and Space Science, vol. 364, p. 50, 2019.

[50] M. Visser, "Rastall gravity is equivalent to Einstein gravity," Physics Letters B, vol. 782, p. 83, 2018.

[51] M. Darabi, H. Moradpour, I. Licata, Y. Heydarzade, and C. Corda, "Einstein and Rastall theories of gravitation in comparison," European Physical Journal C, vol. 78, p. 25, 2018.

[52] G. Darmois, Memorial des sciences mathematiques XXV, Fasticule XXV, Gauthier-Villars, Paris, France, 1927.

[53] W. Israel, "Singular hypersurfaces and thin shells in general relativity," Il Nuovo Cimento B Series, vol. 44, p. 1, 1966.

[54] C. Chirenti and L. Rezzolla, "Gravitational waves from neutron star mergers and their relation to the nuclear equation of state," Physical Review D, vol. 94, Article ID 084016, 2016.

[55] H. M. Yesuf and L. C. Ho, "Gas content regulates the lifecycle of star formation and black hole accretion in galaxies," 2020, http://arxiv.org/abs/2007.12026v1. 